\title{
Unravelling the role of inelastic tunneling into pristine and defected graphene
}

\author{
Palsgaard, Mattias Lau Nøhr; Andersen, Nick Papior; Brandbyge, Mads
}

\section{Published in:}

Physical Review B

Link to article, DOI:

10.1103/PhysRevB.91.121403

Publication date:

2015

Document Version

Publisher's PDF, also known as Version of record

Link back to DTU Orbit

Citation (APA):

Palsgaard, M. L. N., Andersen, N. P., \& Brandbyge, M. (2015). Unravelling the role of inelastic tunneling into pristine and defected graphene. Physical Review B, 91(12), 121403.

https://doi.org/10.1103/PhysRevB.91.121403

\section{General rights}

Copyright and moral rights for the publications made accessible in the public portal are retained by the authors and/or other copyright owners and it is a condition of accessing publications that users recognise and abide by the legal requirements associated with these rights.

- Users may download and print one copy of any publication from the public portal for the purpose of private study or research.

- You may not further distribute the material or use it for any profit-making activity or commercial gain

- You may freely distribute the URL identifying the publication in the public portal 


\title{
Unravelling the role of inelastic tunneling into pristine and defected graphene
}

\author{
Mattias L. N. Palsgaard, Nick P. Andersen, and Mads Brandbyge* \\ Center for Nanostructured Graphene, Department of Micro- and Nanotechnology, Technical University of Denmark, Ørsteds Plads, \\ Building 345E, DK-2800 Kongens Lyngby, Denmark
}

(Received 11 October 2014; revised manuscript received 17 February 2015; published 5 March 2015)

\begin{abstract}
We present a first principles method for calculating the inelastic electron tunneling spectroscopy (IETS) on gated graphene. We reproduce experiments on pristine graphene and point out the importance of including several phonon modes to correctly estimate the local doping from IETS. We demonstrate how the IETS of typical imperfections in graphene can yield characteristic fingerprints revealing, e.g., adsorbate species or local buckling. Our results show how care is needed when interpreting scanning tunneling microscopy images of defects due to suppression of the elastic tunneling on graphene.
\end{abstract}

DOI: 10.1103/PhysRevB.91.121403

PACS number(s): 63.22.Rc, 63.20.dk, 68.37.Ef, 72.10.Di

Imperfections such as lattice defects, edges, and impurity/dopant atoms can degrade the superb transport properties of graphene [1-4], or may, if controlled, lead to new functionality [5]. Scanning tunneling microscopy and spectroscopy (STM/STS) have been used extensively to obtain insights into the local electronic structure of graphene with atomic resolution [6-10]. However, contrary to most STM/STS experiments where elastic tunneling plays the dominant role, for graphene the inelastic tunneling prevails. This was clearly demonstrated experimentally as a "giant" signal in the second derivative of the current with regard to voltage obtained in inelastic electron tunneling spectroscopy (IETS) performed on gated, pristine graphene with STM [6-8]. The pronounced inelastic features are rooted in the electronic structure of graphene. The electrons have to enter the Dirac points corresponding to a finite in-plane momentum leading to weak elastic tunneling. The IETS signal of pristine graphene has been reproduced qualitatively by Wehling et al. by considering the change in the wave-function decay when displacing the carbon atoms along a selected frozen zone-boundary out-of-plane phonon [11]. In general, the important role of the inelastic process complicates the interpretation of STM results on graphene. Ideally, STM images on graphene structures should be accompanied by local STS/IETS measurements, in order to distinguish between contributions from the inelastic and elastic channel. On the other hand, first principles calculations based on density functional theory (DFT) often provide essential unbiased insights into STM/STS/IETS experiments to help the interpretation.

In this Rapid Communication we present a method for DFT calculations of the STS/IETS on gated graphene. We demonstrate its predictive power by reproducing from first principles the features of the experimental results for the giant inelastic conductance of gated pristine graphene [6-8]. We then provide results for IETS signals of defected graphene systems by determining the relative impact on the current of the various phonon modes. In particular, we identify inelastic fingerprints of selected defects, suggesting that IETS measurements can be a powerful tool in the characterization of imperfect graphene. Our analysis also illustrates how one should keep in mind

*mads.brandbyge@nanotech.dtu.dk the in-plane momentum conservation when performing STM on graphene. In particular, we demonstrate how defects can locally lift the suppression of elastic tunneling. The resulting increased local conductance may be misinterpreted as a high local density of states (LDOS).

Method. The calculations are performed with DFT using the SIESTA/TRANSIESTA [12,13] code and the INELASTICA package for inelastic transport [14]. Our system, shown in Fig. 1, is divided into a top lead (source), device and sample lead (drain) following the standard transport setup [13-15]. We consider a suspended graphene sheet located $5 \AA$ below the tip of a gold STM probe model and a voltage bias between the tip and sample leads. The electron-phonon coupling $\left(\mathbf{M}^{\lambda}\right)$ is calculated in the coupling region (green+black atoms in Fig. 1) of phonon modes (index $\lambda$ ) calculated in a dynamical region (black atoms) as described in Ref. [14]. Floating orbitals are included between the STM tip and the graphene sample, to give a better description of the vacuum [16].

Following the lowest order expansion (LOE) [17], simplified and efficient expressions for the IETS signals can be derived under the assumption of weak electron-phonon coupling. The LOE expressions involve just the evaluation of the spectral density matrices for states coming from the tip/sample, $\mathbf{A}_{T / S}(\varepsilon)$, at a discrete number of chemical potentials, $\varepsilon=\mu_{T}, \mu_{S}$, corresponding to the threshold voltage bias $\left(V_{b}\right)$ for excitation of a given phonon $(\lambda),\left|\mu_{T}-\mu_{S}\right|=$ $\hbar \omega_{\lambda}$. Thus the LOE expression does not per se reflect changes in the DOS above the phonon excitation threshold. However, in the context of STS on gated graphene, this is highly relevant since the DOS behavior leads to a distinct dip in the differential conductance at a specific applied voltage, $V_{b}=V_{D}$ [6], enabling a determination of the local chemical potential of graphene.

In order to encompass this important variation in the DOS above threshold we make the following observations (see also Fig. 2). The expressions for the current which gives rise to inelastic signals have a Fermi's golden-rule-like form at low temperature,

$$
\begin{aligned}
I_{i} \approx & \frac{e}{\hbar} \Theta\left(e\left|V_{b}\right|-\hbar \omega_{\lambda}\right) \\
& \times \int_{\mu_{S} \pm \hbar \omega_{\lambda}}^{\mu_{T}} d \varepsilon \operatorname{Tr}\left[\mathbf{M}_{\lambda} \tilde{\mathbf{A}}_{T}(\varepsilon) \mathbf{M}_{\lambda} \mathbf{A}_{S}\left(\varepsilon \mp \hbar \omega_{\lambda}\right)\right],
\end{aligned}
$$




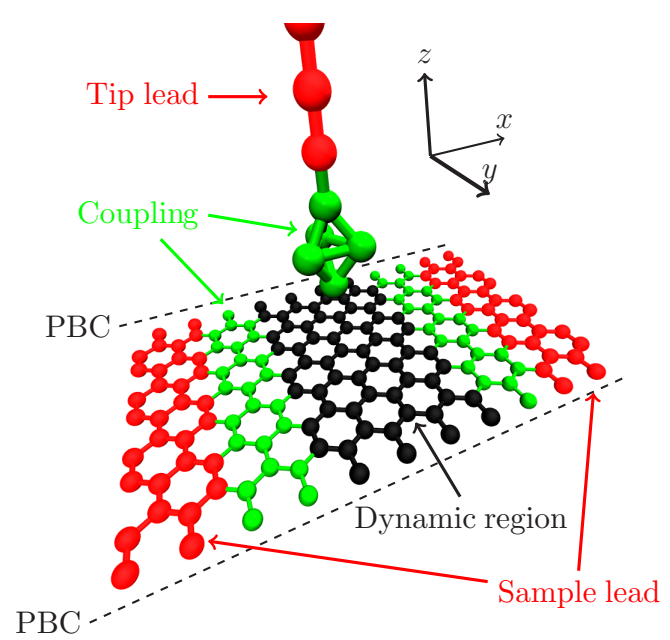

FIG. 1. (Color online) The system setup with semi-infinite leads (red), device region (green), dynamic region (black), and periodic boundary conditions along the dashed lines.

for $V_{b} \gtrless 0$, where $\Theta$ is the Heaviside step function and $\tilde{\mathbf{A}}_{T}$ is the time reversed $\mathbf{A}_{T}$. Above threshold $\left(\left|V_{b}\right|>\hbar \omega_{\lambda}\right)$ the step behavior is unimportant and we are left with the bias behavior of the integral. For finite bias the states in the device, that is, the spectral functions, change with $V_{b}$. However, in this STM setup, the device is strongly coupled to the sample lead and very weakly coupled to the tip lead. Consequently, the potential in the device is pinned to that of the sample lead, which is the Fermi level $\varepsilon_{F}$ of graphene. The DOS of the gold STM probe varies slowly with regard to energy. Thus the only important voltage dependent term in Eq. (1) is the tip chemical potential defined by $\mu_{T}=\varepsilon_{F}+e V_{b}$, with $\mu_{S}=\varepsilon_{F}$, yielding the differential conductance expression

$$
\partial_{V_{b}} I_{i} \approx \gamma_{\lambda} \partial_{V_{b}} \mathcal{I}^{\text {sym }},
$$

where

$$
\gamma_{\lambda}=\operatorname{Tr}\left[\mathbf{M}_{\lambda} \tilde{\mathbf{A}}_{T}\left(e V_{b}+\varepsilon_{F}\right) \mathbf{M}_{\lambda} \mathbf{A}_{S}\left(e V_{b}+\varepsilon_{F} \mp \hbar \omega_{\lambda}\right)\right],
$$

for $V_{b} \gtrless 0$, and $\mathcal{I}^{\text {sym }}$ is a temperature broadened version of the step function in Eq. (1) [14]. Equation (2) is equivalent to the usual LOE expression but valid above threshold due to the constant tip DOS. The same argument can be applied to the

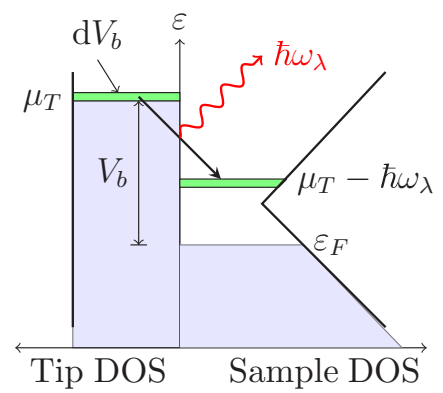

FIG. 2. (Color online) Energy diagram showing the important (green) contributions of the tip/sample DOS when calculating the differential conductance for $V_{b}>\hbar \omega_{\lambda}$.
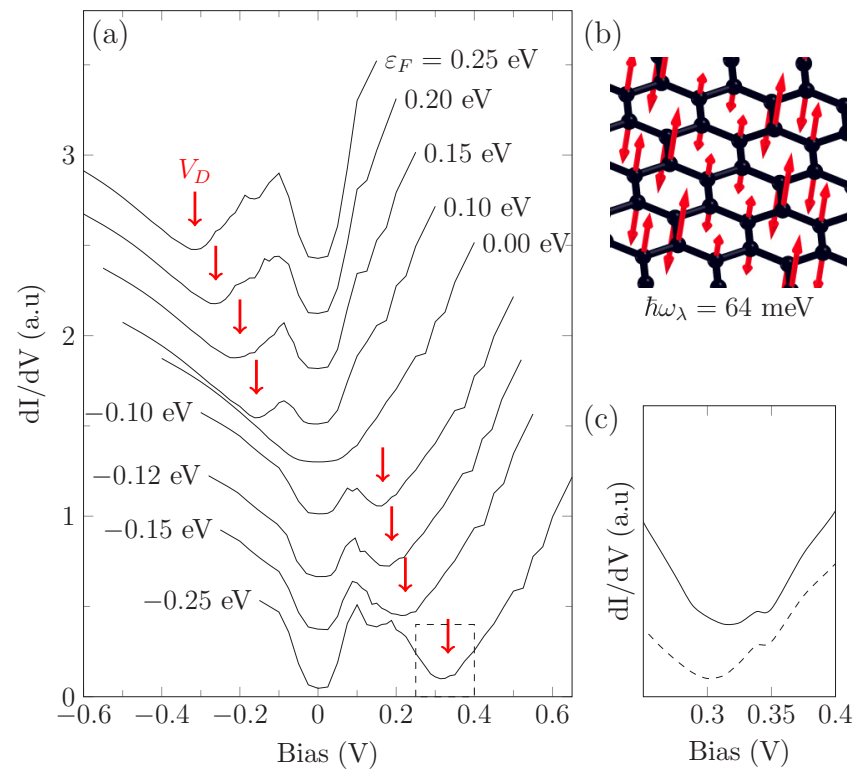

FIG. 3. (Color online) (a) Calculated STS spectra of pristine graphene at different Fermi levels. (b) Out-of-plane acoustic graphene phonon. (c) Closeup of the dip at $V_{D}$ for $\varepsilon_{F}=-0.25 \mathrm{eV}$, when including only phonons in the $0-400 \mathrm{meV}$ (solid) and $60-70 \mathrm{meV}$ (dashed) energy range.

other terms in LOE. See supplemental material [18] for details of the calculation used, to obtain STS spectra.

Results-pristine graphene. Calculated STS spectra on pristine graphene for a number of different applied gate voltages (Fermi levels) are shown in Fig. 3(a). The gap feature around $V_{b}=0$ of width $0.13 \mathrm{~V}$ is reproduced in detail and the dip at $V_{D}$, caused by inelastic tunneling into the charge neutrality point of graphene, appears outside the gap, as seen in experiments [6-8]. As the gate is applied, $V_{D}$ moves across the spectrum, changing polarity, while the position and width of the gap feature is stable.

Most major steps in differential conductance come from acoustic out-of-plane phonons at energies just below $67 \mathrm{meV}$ [19]. In particular, the mode shown in Fig. 3(b) gives a large contribution. However, we find that acoustic out-of-plane graphene phonons with energies as low as $42 \mathrm{meV}$ give considerable contributions as well. We also find important inelastic signals from optical graphene phonons at energies above $67 \mathrm{meV}$. The additional features away from $67 \mathrm{mV}$ make up about half the signal, and have not been included in previous studies [11]. If we restrict our calculations to phonons in the $60-70 \mathrm{meV}$ range, we obtain a $15 \mathrm{mV}$ change in $V_{D}$ [see Fig. 3(c)] and changes in both the width and height of the inelastic gap. The change in $V_{D}$ is caused mostly by the experimentally observed [8] inelastic signal near $150 \mathrm{mV}$, coming from the optical in-plane modes, and occurs for $\left|V_{D}\right|>150 \mathrm{mV}$. In STS experiments $V_{D}$ is used to extract the energy position of the charge neutrality point from $E_{D}=e\left|V_{D}\right|-\hbar \omega_{0}$, where $\hbar \omega_{0}=63 \mathrm{meV}$ is half the width of the gap feature which corresponds to the energy of an acoustic out-of-plane graphene phonon [6,7]. The change in $V_{D}$ could explain why all points with $\left|E_{D}\right|<100 \mathrm{meV}$ in the $E_{D}$ versus gate voltage plot of Ref. [7] fall below the fitted line. The local 


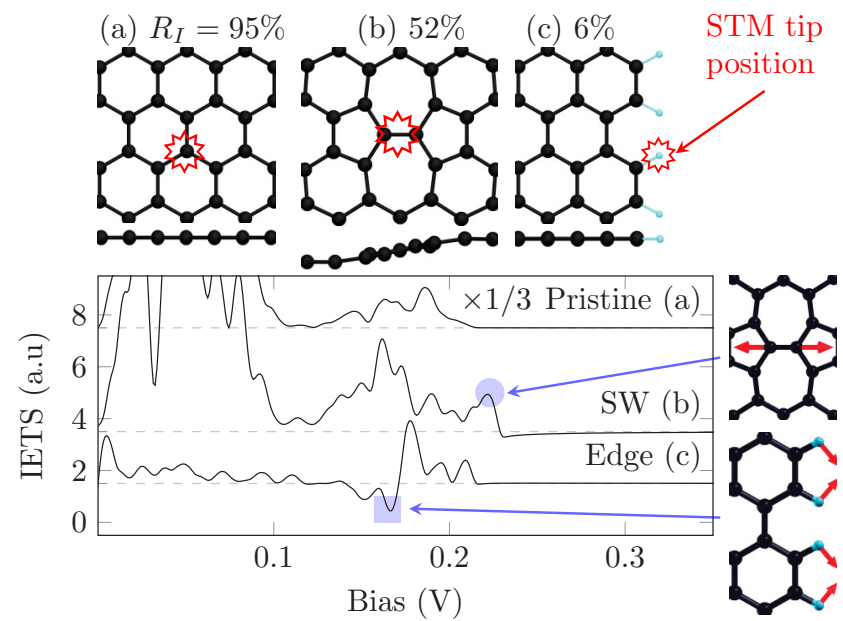

FIG. 4. (Color online) IETS as a function of bias for pristine graphene, a Stone-Wales defect, and a hydrogen passivated armchair edge (geometries shown above the plot). The blue marker indicates characteristic signals. The fraction of differential conductance coming from the inelastic channel $\left(R_{I}\right)$ is shown above the geometries.

charge-carrier density $(n)$ of graphene is also extracted from $V_{D}$ in STS experiments [7,9,10]. Mistaking $E_{D}=100 \mathrm{meV}$ for $E_{D}=115 \mathrm{meV}$ results in a $32 \%$ error in $n$. To capture these experimental details one must include several phonons, and account for their impact in an ab initio manner.

Encouraged by the agreement for pristine graphene, we next predict the inelastic signals from various defects to shed light on what information can be obtained from STM-IETS.

Results-structurally defected graphene. In Fig. 4 we show the calculated IETS spectra from an on-top position in pristine graphene [Fig. 4(a)], directly above a Stone-Wales defect (SW) [Fig. 4(b)], and above a passivated armchair edge [Fig. 4(c)]. The result shown for pristine graphene is the same at hollow sites and bridge sites. The gap feature shows up as giant peaks in the IETS below $100 \mathrm{mV}$ for pristine graphene. The signals from in-plane graphene phonons are also seen at $150 \mathrm{mV}$ and above. We find that the low voltage IETS above a SW are very similar to that of pristine graphene. Gap like features have also been observed experimentally for regions with heptagon-pentagon defects [20]. However, a characteristic signal can be seen at $V_{b}=223 \mathrm{mV}$ bias, above any of the pristine graphene phonon bands which can be traced to the high-frequency stretch mode localized at the twisted C-C bond shown in Fig. 4.

Ignoring the out-of-plane buckling introduced to the graphene sheet near a SW, and calculating the IETS for a flat SW system, leads to a $5 \mathrm{mV}$ blueshift of the signal from the twisted C-C bond, as previously proposed [21]. Furthermore, signals from in-plane modes at $150 \mathrm{mV}$ in Fig. 4(b) generally become weaker in the flat system, indicating a weaker coupling of these modes to the out-of-plane current. We also see strong signals at low bias. These signals are caused by low-frequency sinelike out-of-plane modes. These modes couple strongly to the current because they break the mirror symmetry across the twisted C-C bond. In the buckled system, this symmetry is inherently broken, leading to an increase in elastic tunneling. Measuring strong low bias inelastic signals and a $228 \mathrm{mV}$
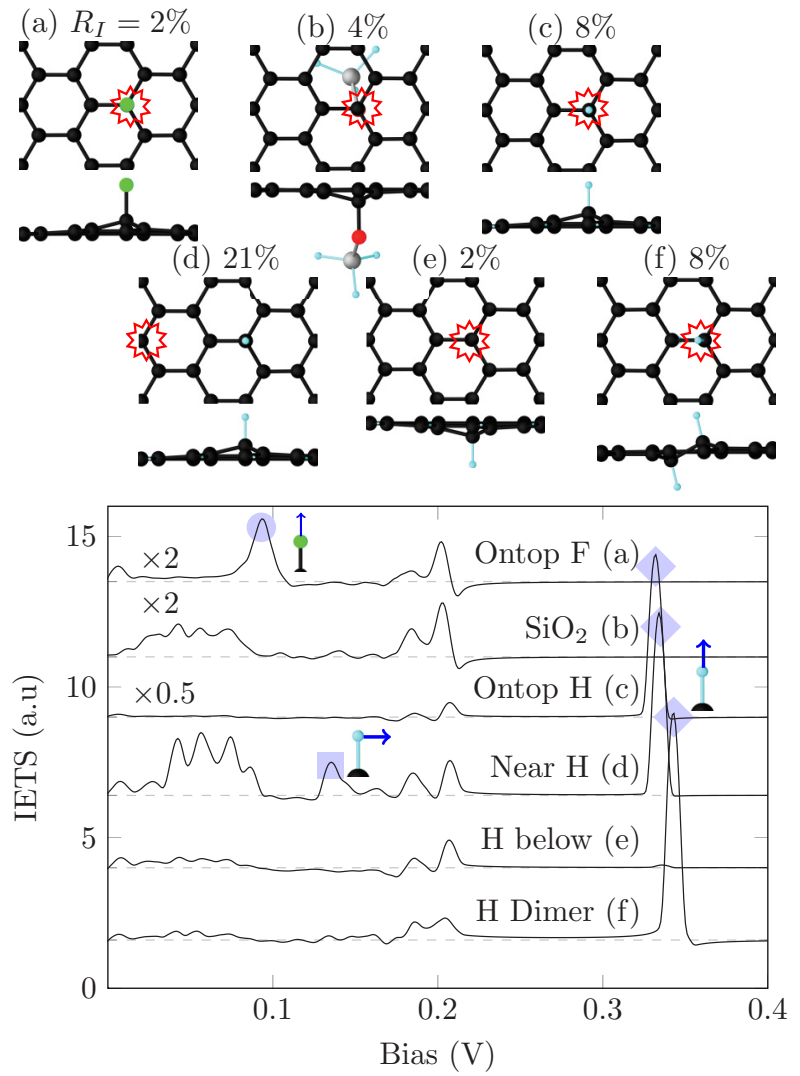

FIG. 5. (Color online) IETS as a function of bias voltage, for various adsorbates on graphene (geometries shown above the plot). Fingerprints for each adsorbate are marked and the phonon shown. The fraction of differential conductance coming from the inelastic channel $\left(R_{I}\right)$ is shown above the geometries.

signal above a SW therefore indicates that it is in a metastable flat configuration, whereas increased elastic transmission and a $223 \mathrm{mV}$ signal is a sign of local buckling. Above a passivated armchair edge, a dip in IETS is seen at $V_{b}=168 \mathrm{mV}$, which is caused by a collective transverse mode of the hydrogen atoms shown in Fig. 4. Changing the mass of the passivating agent to that of fluor, we observe a corresponding change in the position of the inelastic signals. This indicates that IETS can be used to obtain knowledge of graphene edge passivation.

Results-adsorbates on graphene. In Fig. 5 we show IETS spectra from a range of different covalently bonded impurities. For all systems, delocalized in-plane graphene modes cause signals around $200 \mathrm{mV}$. In Fig. 5(a) a clear inelastic signal from the longitudinal mode of a fluor adsorbate is seen at 95 $\mathrm{mV}$. Above a hydrogen adsorbate, we see a strong inelastic peak at $332 \mathrm{mV}$ caused by the stretch mode of the C-H bond [see Fig. 5(c)]. This signal serves as a fingerprint for a hydrogen impurity above the graphene sheet as opposed to below where the signal disappears, as can be seen in Fig. 5(e). The corresponding STS spectra show a strong zero-energy peak [22]; this behavior is, however, expected for all covalently bonded impurities [23], above or below the sheet, and therefore cannot be used as a fingerprint.

The STS spectra on the hydrogenated system with the probe above a carbon atom $4.25 \AA$ laterally away from the 
impurity in Fig. 5(d) show additional signals. The graphene out-of-plane phonon signals reappear and a signal is also seen at $134 \mathrm{mV}$, caused by a transverse mode of the $\mathrm{C}-\mathrm{H}$ bond. Above a graphanelike hydrogen dimer [Fig. 5(f)] the signal caused by the $\mathrm{C}-\mathrm{H}$ bond stretch mode is seen, however, here it is caused by two degenerate modes and blueshifted by $11-16 \mathrm{mV}$, indicating a lower energy configuration.

Common for all the imperfect systems is that the gap seen in pristine graphene is quenched and considerable elastic tunneling is seen, as indicated by the severe reduction of the inelastic conductance ratio $\left(R_{I}\right)$ in Figs. 4 and 5. Out-of-plane corrugations in the graphene sheet can lift the suppression of elastic tunneling if they are on the same length scale as the graphene lattice constant [11]. Our results indicate that defects can also lift the suppression locally. This is because the selection rules causing the suppression in pristine graphene are a result of the translational symmetry of the crystal lattice. When this symmetry is broken, the suppression is lifted and the elastic tunneling dominates. The expected order of magnitude change in tunneling conductance should lead to bright spots in STM topographies. In the case of granular chemical vapor deposited (CVD) graphene, protruding grain boundaries are often attributed to localized electronic states [20]. Our results here point out that one may expect increased tunneling near disordered areas of graphene, even if no localized electronic states are present and the area is completely flat. As seen in Figs. 5(b) and 5(e), this is also the case for strong interactions with a $\mathrm{SiO}_{2}$ substrate or hydrogen sitting below the graphene sheet, which should therefore be visible as protruding from the graphene sheet.

In summary, we have presented a first principles method and used it for calculations of IETS and STS spectra of pristine and defected graphene. We showed how measured STS spectra on pristine gated graphene can be reproduced in detail as a function of gating. The inclusion of several phonons had a strong impact on all aspects of the STS spectrum of pristine graphene. In particular, we found that including optical in-plane phonons changed the $V_{D}$ value for certain gate voltages. This is of importance for studies where IETS is used to probe the local doping of graphene $[7,9,10]$ where it may lead to a significant overestimation of the local charge inhomogeneity. We predicted the IETS of typical imperfections in graphene, and demonstrated how these can yield characteristic fingerprints revealing, e.g., adsorbate species or local buckling. Additional elastic contributions above defects should make them protrude in STM regardless of the actual geometric or electronic structure, and care is needed when interpreting STM images.

We gratefully acknowledge discussions with Thomas Frederiksen, Aran Garcia-Lekue, and Rasmus Bjerregaard Christensen. The Center for Nanostructured Graphene (CNG) is sponsored by the Danish Research Foundation, Project DNRF58.
[1] A. Geim and K. S. Novoselov, Nat. Mater. 6, 183 (2007).

[2] A. H. C. Neto, F. Guinea, N. M. R. Peres, K. S. Novoselov, and A. K. Geim, Rev. Mod. Phys. 81, 109 (2009).

[3] K. S. Novoselov, A. K. Geim, S. V. Morozov, D. Jiang, Y. Zhang, S. V. Dubonos, I. V. Grigorieva, and A. A. Firsov, Science 306, 666 (2004).

[4] A. C. Ferrari, J. C. Meyer, V. Scardaci, C. Casiraghi, M. Lazzeri, F. Mauri, S. Piscanec, D. Jiang, K. S. Novoselov, S. Roth, and A. K. Geim, Phys. Rev. Lett. 97, 187401 (2006).

[5] J. G. Pedersen, T. Gunst, T. Markussen, and T. G. Pedersen, Phys. Rev. B 86, 245410 (2012).

[6] Y. Zhang, V. W. Brar, F. Wang, C. Girit, Y. Yayon, M. Panlasigui, A. Zettl, and M. Crommie, Nat. Phys. 4, 627 (2008).

[7] R. Decker, Y. Wang, V. W. Brar, W. Regan, H. Z. Tsai, Q. Wu, W. Gannett, A. Zettl, and M. F. Crommie, Nano Lett. 11 (2011).

[8] V. W. Brar, S. Wickenburg, M. Panlasigui, C.-H. Park, T. O. Wehling, Y. Zhang, R. Decker, C. Girit, A. V. Balatsky, S. G. Louie, A. Zettl, and M. F. Crommie, Phys. Rev. Lett. 104, 036805 (2010).

[9] P. Cao, J. O. Varghese, K. Xu, and J. R. Heath, Nano Lett. 12, 1459 (2012).

[10] S. J. Goncher, L. Zhao, A. N. Pasupathy, and G. W. Flynn, Nano Lett. 13, 1386 (2013).

[11] T. O. Wehling, I. Grigorenko, A. I. Lichtenstein, and A. V. Balatsky, Phys. Rev. Lett. 101, 216803 (2008).
[12] J. M. Soler, E. Artacho, J. D. Gale, A. García, J. Junquera, P. Ordejón, and D. Sánchez-Portal, J. Phys.: Condens. Matter 14, 2745 (2002).

[13] M. Brandbyge, J. L. Mozos, P. Ordejón, J. Taylor, and K. Stokbro, Phys. Rev. B 65, 165401 (2002).

[14] T. Frederiksen, M. Paulsson, M. Brandbyge, and A. P. Jauho, Phys. Rev. B 75, 205413 (2007).

[15] We use a split polarized double zeta (DZP) basis set, a mesh cutoff of 200 Ry, a Monkhorst-Pack $k$-point mesh of $1 \times 2 \times 1$, and the local density approximation (LDA) exchange-correlation (xc) functional [24] to calculate the electronic structure. A supercell dimension ( $k_{y}$ points) of $27 \AA \times 12.8 \AA(101) / 34.5 \AA \times$ $25.5 \AA(\Gamma) / 49.3 \AA \times 12.8 \AA(\Gamma) / 27 \AA \times 17 \AA(\Gamma)$ is used for inelastic transport for the pristine/SW/edge/adsorbate configuration.

[16] A. Garcia-Lekue and L. W. Wang, Phys. Rev. B 82, 035410 (2010).

[17] J. T. Lü, R. B. Christensen, G. Foti, T. Frederiksen, T. Gunst, and M. Brandbyge, Phys. Rev. B 89, 081405 (2014).

[18] See Supplemental Material at http://link.aps.org/supplemental/ 10.1103/PhysRevB.91.121403 for convergence tests and details of the STS calculation including above excitation threshold DOS effects.

[19] M. Mohr, J. Maultzsch, E. Dobardzic, S. Reich, I. Milosevic, M. Damnjanovic, A. Bosak, M. Krisch, and C. Thomsen, Phys. Rev. B 76, 035439 (2007). 
[20] L. Zhao, M. Levendorf, S. Goncher, T. Schiros, L. Paova, A. Zabet-Khosousi, K. T. Rim, C. Gutierez, D. Nordlund, C. Jaye, M. Hybertsen, D. Reichman, G. W. Flynn, J. Park, and A. N. Pasupathy, Nano Lett. 13, 4659 (2013).

[21] J. Ma, D. Alfe, A. Michaelides, and E. Wang, Phys. Rev. B 80, 033407 (2009).
[22] M. Scheffler, D. Haberer, L. Petaccia, M. Farjam, R. Schlegel, D. Baumann, T. Hänke, A. Gruneis, M. Knupfer, C. Hess, and B. Buchner, ACS Nano 6, 10590 (2012).

[23] T. O. Wehling, M. I. Katsnelson, and A. I. Lichtenstein, Phys. Rev. B 80, 085428 (2009).

[24] J. P. Perdew and A. Zunger, Phys. Rev. B 23, 5048 (1981). 